\title{
Les lésions moléculaires des types 1 et 2 du syndrome de Waardenburg
}

Le syndrome de Waardenburg (SW) est une affection autosomique dominante, comportant une surdité de gravité variable, des anomalies pigmentaires (mèche blanche, hétérochromie de l'iris), et une morphologie faciale modifiée. Il en existe deux types cliniques, la différence principale étant l'existence, dans le type 1, d'un déplacement latéral de l'angle interne de l'œil (dystopia canthorum). Une variante comporte des anomalies des membres, constituant un type 3 qu'on rattache au type 1. En 1992 a été décrite une localisation précise du gène du type 1 en $2 q 35$, sur un gène humain homologue du gène pax-3 de la souris, qui code pour un facteur de transcription exprimé d'abord dans les précurseurs de la crête neurale. Ce gène est délété dans la mutation splotch de la souris, dont on connaît aujourd'hui plusieurs allèles. Une brève de médecine/sciences ( ${ }^{\circ} 4$, vol. 8, p. 393) relatait ces faits, ainsi que les deux premières mutations du gène humain $P A X-3$. Un consortium de 23 auteurs [1] fait le point actuel sur l'hétérogénéité génétique du SW. Il confirme que seul le type 1 (avec le type 3 qui s'y rattache) est lié au gène $P A X-3$. Sur un total de 49 familles interprétables, 25 sont liées ; dans 24 de ces familles, la lésion moléculaire a pu être identifiée. Tous les types d'anomalies s'y rencontrent: délétions étendues ou limitées, erreurs d'épissage, mutations faux-sens surtout dans l'exon 2. Chaque malade possédait une mutation différente. Le travail du consortium montre bien par ailleurs que le SW2 relève d'une autre origine génétique, mais il ne la précise pas. La nature de celle-ci vient d'être élucidée par une équipe britannique de Manchester [2].

L'origine de cette découverte réside dans des travaux effectués sur les mutants mi (microphthalmia) de la souris, dont on connaît actuellement 17 allèles, porteurs d'anomalies oculaires et cutanées. Ce gène a été cloné en 1993 [3] ; il code pour une protéine à hélice-boucle-hélice et glissière à leucine, de 419 acides aminés, ressemblant à plusieurs facteurs de transcription. Le gène contient 9 exons. Le gène homologue humain MITF (microphthalmia-associated transcription factor) a été localisé sur le chromosome 3 en 3pl2-pl4 [4] et cloné [5]. Deux familles ont pu jusqu'à présent avoir leur mutation analysée. La première porte un changement $A G \rightarrow$ CG affectant le site accepteur du début de l'exon 5 , ce qui devrait éliminer ce dernier, et créer un décalage avec terminaison au codon 211. Dans la deuxième famille, la suppression d'un site donneur GT à la fin de l'exon 1 devrait entraîner une terminaison dans l'intron 1,18 codons plus loin, avec un produit de 29 acides aminés. Quatre autres sujets montrent des anomalies de certaines bandes mais sont encore en cours d'examen.

L'interprétation des mécanismes qui sous-tendent ces deux affections dominantes soulève les problèmes habituels, avec peut-être des difficultés particulières. Nous rappellerons une fois de plus que des interprétations opposées peuvent rendre compte d'une maladie dominante: une perte de fonction d'une protéine anormale, qui empêche le fonctionnement de l'allèle normal par interaction ; ou un gain de fonction, rendant une protéine active en permanence ou lui conférant des propriétés nouvelles. Qu'en est-il dans le SW ?

Les mutations des souris splotch et $m i$ sont le plus souvent semi-dominantes : les homozygotes sont beaucoup plus gravement atteints et souvent non viables. Parmi les mutants $m i$, certains sont même récessifs. La gravité clinique semble dépendre de la lésion moléculaire ; mais chez les hétérozygotes une demi-dose ne paraît pas suffire : les mutants $s p r$ (por- tant une délétion) ou $s p$ (anomalie de l'épissage) sont plus sévèrement atteints que $s p d$ (splotch delayed, mutation faux-sens).

Chez l'homme, les deux cas de SW2 élucidés donnent nécessairement des protéines tronquées qui ne sont sans doute pas fonctionnelles, en particulier celle qui n'aurait que 29 acides aminés. Les anomalies multiples trouvées dans le type l comportent des délétions, insertions et épissages anormaux; il ne s'agit donc pas, dans la plupart de ces cas, de protéines ayant acquis des fonctions nouvelles. Il est vrai que la gravité est variable selon les familles, mais une corrélation solide n'a pas encore été établie entre les symptômes et les lésions moléculaires; les auteurs soulèvent, pour certains cas, l'hypothèse d'épissages alternatifs qui rétabliraient une phase de lecture, hypothèse qui ne nous paraît pas pouvoir être généralisée. Le mystère du mécanisme de fonctionnement de ces facteurs de transcription et de leurs anomalies reste encore à percer.

J.C.D.

1. Farrer LA, Arnos KS, Asher JH Jr, et al. Locus heterogeneity for Waardenburg syndrome is predictive of clinical subtypes. Am J Hum Genet 1994 ; $55: 728-37$.

2. Tassabehji M, Newton VE, Read AP. Waardenburg syndrome type 2 caused by mutations in the human microphthalmia (MITF) gene. Nature (jpnet $1994 ; 8: 251-5$.

3. Hodgkinson CA, Moore KJ, Nakayama A, Steingrimsson E Copeland MC; Jenkins NA, Amheiter H. Mutations at the mouse microphtalmia locus are associated with defects in a gene encoding a novel basic-helix-zipper protein. Cell 1993; 74 : 395-404.

4. Hughes A, Newton VE, Liu XZ, Read AP. A gene for Waardenburg syndrome type 2 maps close to the human homologue of the microphtalmia gene on chromosome 3 pl2-pl4. Nature Genet $1994 ; 7: 509-12$.

5. Tashibana M, Perez-Jurado LA, Nakayama A, et al. Cloning of MITF, the human analogue of the mouse microphtalmia gene and assignment to chromosome 3pl4.1-pl2.3. Hum Mol Genet 1994; $3: 553-7$. 